\title{
Roles of Tet2 in meiosis, fertility and reproductive aging
}

\section{Dear Editor,}

Ten-eleven translocation (Tet) enzymes play important roles in DNA demethylation involved in various biological processes including stem cell pluripotency and differentiation, and tumorigenesis (Dawlaty et al., 2013; Wu and Zhang, 2017). Tet2 deficiency or mutation leads to severe hematopoietic defects or myeloid malignancies in mice (Delhommeau et al., 2009; Ko et al., 2010; Moran-Crusio et al., 2011). Restoration of TET2 blocks aberrant self-renewal and leukemia progression in patients possessing TET2 mutations (Cimmino et al., 2017). DNA methylationbased biomarkers, or "epigenetic clocks", link developmental and maintenance processes to biological aging (Horvath and Raj, 2018). Interestingly, age-associated TET2 mutations have been found to drive myeloid dysfunction, cancer and cardiovascular disease (review (Ferrone et al., 2020)). Nevertheless, there has been lack of direct evidence demonstrating that Tet2 mutations or deficiency can actually accelerate aging.

Reproductive aging particularly in female germline predates the aging of somatic organs in the body. The ovaries play an important role in maintaining the normal function of the female reproductive system and hormone secretion. Ovarian aging is mainly characterized by a sharp decrease in the number of oocytes and follicles as well as declined oocyte quality. Loss of Tet1 causes meiosis defects in fetal ovaries and in adult testis (Huang et al., 2020; Yamaguchi et al., 2012), and reduces oocyte number (Yamaguchi et al., 2012). Here we show that Tet2 plays multiple roles during meiosis and oocyte development. Moreover, Tet2 deficiency does not affect oocyte number, unlike Tet1 (Yamaguchi et al., 2012), but noticeably delays meiotic progression and reduces oocyte quality and mouse fertility, accelerating age-associated infertility.

To study whether Tet2 regulates fertility with age, we employed Tet2-deficient mice generated by deleting exon 3 (See methods). Homozygous mutant mice $\left(\mathrm{Tet}^{-l-}\right)$ were generated by crossing the heterozygous mice. The primers were designed to detect three different genotypes (wild-type (WT) Tet $^{+/+}$, Tet2 $^{+/-}$and Tet2 $^{-/-}$) (Fig. S1A), and the exon 3 deletion in $\mathrm{Tet}^{-/-}$female ovaries was also validated by qPCR of mRNA expression (Fig. S1B). Probably Tet2 protein level is very low in adult ovary, such that Tet2 protein signal could not be detectable. Thus, we confirmed that Tet2 protein was absent in $\mathrm{Tet}^{-{ }^{--}}$blastocysts by immunofluorescence (Fig. S1C). Tet2 ${ }^{-1-}$ mice were born at normal Mendelian ratio (Fig. S1D). The $5 \mathrm{hmC}$ levels were decreased by immunofluorescence in Tet2-deficient oocytes and granulosa cells (Fig. S1E and S1F). Hence, Tet2 knockout decreases the $5 \mathrm{hmC}$ level, as expected.

Tet2 ${ }^{-1-}$ female mice at young, middle-age and old age (specified in the figure legend) after successful mating with young males exhibited reduced fecundity as shown by smaller litter size, compared with age-matched WT females. The litter size reduced by half when the Tet2 $2^{-/-}$mice reached at middle-age, and most old $\mathrm{Tet}^{-/-}$mice failed to give birth to a pup (Fig. 1A). Ovarian weight of WT females increased at middle-age and then decreased at the old age. Ovarian weight of $T e t 2^{-1-}$ mice was generally lighter than that of WT mice at young and middle-age, but did not differ from WT mice at the old age (Fig. S1G and S1H). However, the average number of follicles at various developmental stages in Tet2 ${ }^{-1-}$ ovaries did not differ from that of WT ovaries regardless of age (Fig. S1I and S1J). Therefore, Tet2 deficiency decreases fecundity and accelerates infertility with age, but intriguingly, the infertility is not related to follicle reserve or oocyte numbers.

We assumed that Tet2 deficiency may negatively impact germ cell development and oocyte quality. We initially examined whether Tet2 deficiency affects meiosis in the fetal gonad. Female meiosis undergoes unique long prophase I, including leptotene, zygotene, pachytene and diplotene, followed by arrest at diakinesis during E13.5-E18.5 after PGC specification (Saitou and Miyauchi, 2016). We chose four embryonic days, including E15.5, E16.5, E17.5 and E18.5, to observe meiosis progression. All the four stages in prophase I could be found at the right time in WT meiocytes, but meiosis progression notably was postponed by Tet2 deficiency (Fig. S2A). While nearly $60 \%$ of meiocytes reached pachytene stage at E16.5 in WT meiocytes with clear synapsis indicated by pairing of SYCP 3 and SYCP1 (synaptonemal complex protein $3 / 1$ ), only about $30 \%$ of Tet $^{-/-}$meiocytes entered pachytene and more than $60 \%$ remained at zygotene stage (Fig. S2B). At E18.5, 56\% of WT meiocytes were at diplotene stage, whereas only $18 \%$ 
A

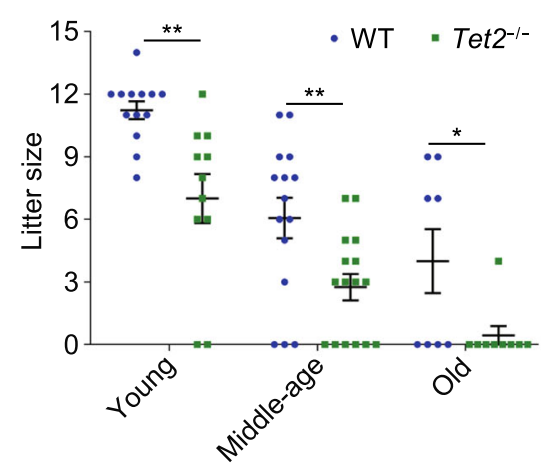

B

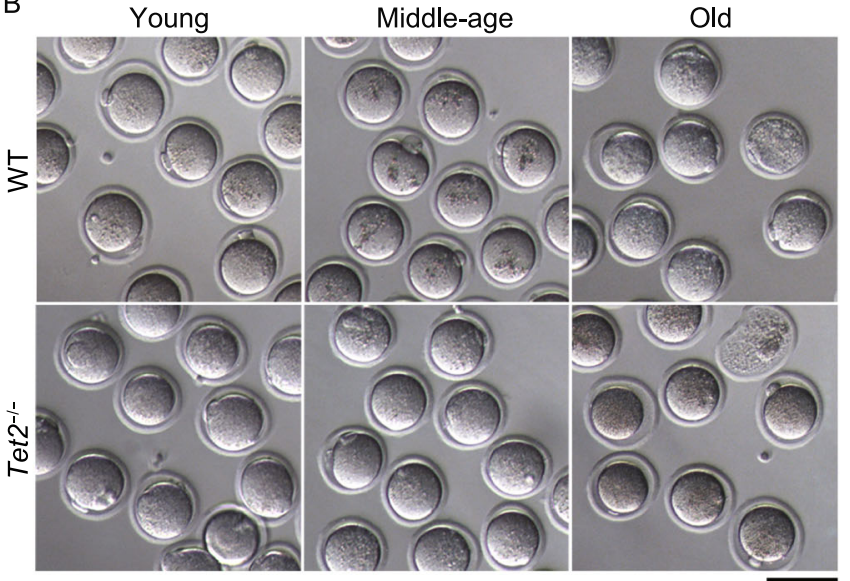

F
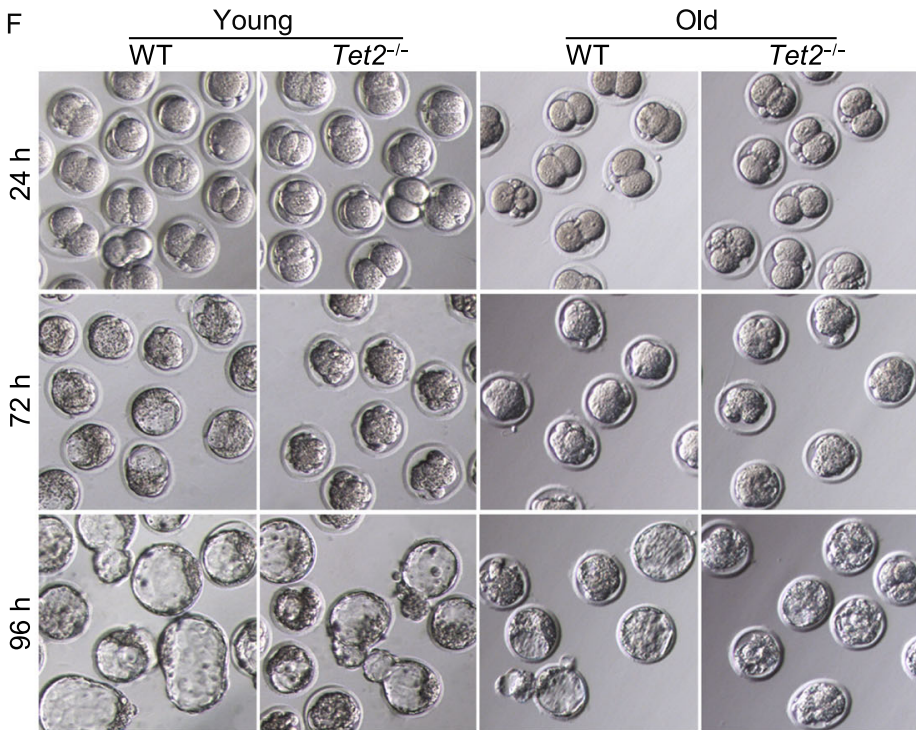

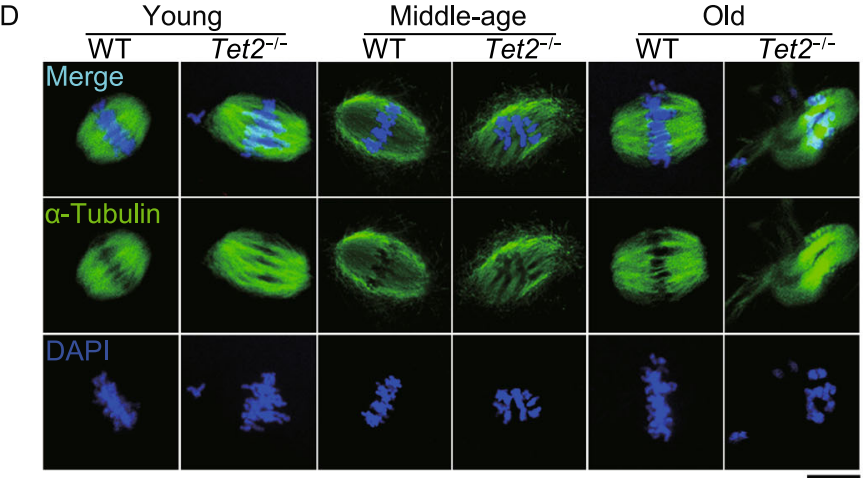

C
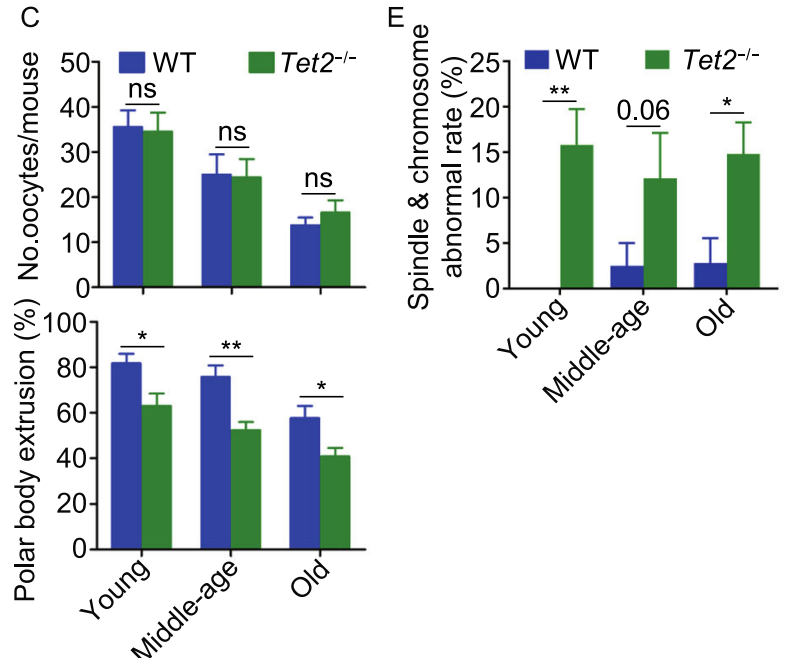

G $\square \mathrm{WT} \square$ Tet2 $^{-1-}$
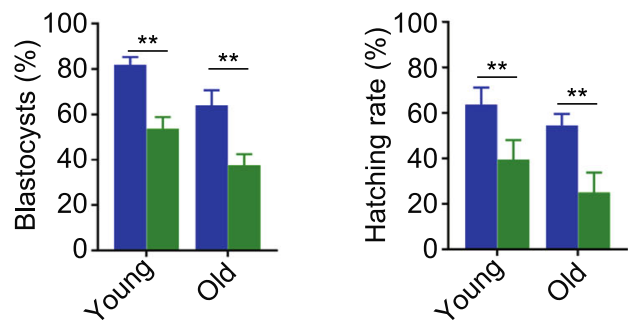
Figure 1. Tet2 knockout causes subfertility and impairs oocyte and early embryo development. (A) Average litter size of wild-type (WT) and Tet2 knockout $\left(\right.$ Tet $\left.2^{-1-}\right)$ female mice at three different ages (young, 2-4 months; middle-age, 7-9 months; old, 10-12 months). $n>10$ for successfully mated female mice for young and middle-age group and $n \geq 8$ mice for old group are pooled. Each spot in round (blue) or square (green) shape represents the number of pups delivered from a successfully mated female mouse. (B) Representative images of superovulated oocytes from WT and $T e t 2^{-/-}$female mice at three different ages. Scale bar, $100 \mu \mathrm{m}$. (C) Comparison of oocyte yield per mouse (top) and percentage of the first polar body extrusion (bottom). $n=4-6$ mice per group. (D) Representative images of oocytes stained with $\alpha$-Tubulin antibody (green) and DAPI (blue) from three different age groups. Scale bar, $10 \mu \mathrm{m}$. (E) Spindle and chromosome abnormal rate between WT and Tet2 ${ }^{-/-}$oocytes from three different age groups. $n=3-4$ mice per group. (F) Morphology of embryo development at 2-cell (24 h), morula (72 h) and blastocyst (96 h) stage following in vitro fertilization of oocytes. Oocytes were obtained from WT and $\mathrm{Tet}^{-/-}$mice from young or old group and sperm from healthy ICR male mice. $(G)$ Percentage of developed blastocysts or blastocyst hatching rate $96 \mathrm{~h}$ after in vitro fertilization from young and old oocytes. $n=3-5$ mice, and about 50 blastocysts were counted for each group. The bars indicate mean \pm SEM. ${ }^{\star} P<0.05$; ${ }^{*} P<0.01$; ns, no significant difference.

reached diplotene and most $(73 \%)$ still were at pachytene stage. These results demonstrated that Tet2 is required for completion of normal meiosis progression at right timing.

To understand the molecular basis underlying role of Tet2 in the initiation of meiosis, we purified germ cells from E13.5 female gonads of Tet2 $^{-1-}$ and WT, and profiled their transcriptome by RNA-seq. We focused on this time point because this is when female PGCs enter meiotic prophase after epigenetic reprogramming (Saitou and Miyauchi, 2016). Important meiotic genes involving meiosis initiation, synapsis formation, crossover and recombination, including Mei1, Ccnb3, Meioc, Sycp1, Sycp2, Msh5, Rad21I and Prdm9, were downregulated in Tet $^{-1-}$ compare to WT germ cells (Fig. S2C). The transcription profile partially interpreted delayed meiosis progression in embryonic gonads resulting from Tet2 deficiency.

The initiating event of meiotic recombination is a programmed DNA double-strand breaks (DSBs) introduced to the genome by Spo11. The DSBs which can be detected by the presence of $\mathrm{YH} 2 \mathrm{AX}$, must be subsequently repaired to form crossovers or noncrossovers. DNA double-strand breaks and repair are required for homologous pairing and synapsis prior to meiotic recombination. Delayed homologous synapsis and meiotic progression might result from defective DNA repair during meiosis. To test this hypothesis, we assayed DNA damage by immunofluorescence assay for
$\mathrm{YH} 2 \mathrm{AX}$ and other key meiosis-associated proteins in the meiocyte spreads prepared from E17.5 gonads. Proportion of $\mathrm{YH} 2 \mathrm{AX}$ positive and partially positive meiocytes increased at pachytene in $\mathrm{Tet}^{-/-}$mice (Fig. S3A-C), suggesting that DSBs are not completely repaired in some $\mathrm{Tet}^{-/-}$meiocytes. RAD51 is a DSB repair-associated recombinase needed for both mitotic and meiotic recombination. Yet, RAD51 foci were not increased in Tet2 ${ }^{-1-}$, compared to WT meiocytes (Fig. S3D and S3E). Late-recombination marker MLH1 normally appears at the designated crossover sites. Number of MLH1 foci also did not differ between Tet2 ${ }^{-1-}$ and WT meiocytes (Fig. S3F and S3G), suggesting that the crossover formation was not impaired. SYCP1/SYCP3-positive staining signal was used to indicate synaptonemal complexes, and 20 synaptonemal complex elements could be found per Tet2 ${ }^{-/-}$meiocyte, like in WT cell (Fig. S3H and S3I). These results suggest that approximately $60 \%$ of $\mathrm{Tet}^{-1-}$ meiocytes can still undergo normal homologous chromosome pairing and meiosis recombination at E17.5.

Next, we asked whether DNA damage could accumulate in Tet2 ${ }^{-1-}$ oocytes compared to that of WT oocytes at later stages. We detected the level of $\mathrm{yH} 2 \mathrm{AX}$ in the ovary at postnatal day (PD) 6 , because almost all of meiocytes developed to primordial follicles at this time. In primordial follicles, more intense $\mathrm{YH} 2 \mathrm{AX}$ signal was found in the nucleus of $\mathrm{Tet}^{-1-}$ than in WT oocytes (Fig. S3J and S3K). Elevated DNA damage indicated by $\mathrm{YH} 2 \mathrm{AX}$ foci was still present in $T e t 2^{-1-} \mathrm{GV}$ oocytes from young and old female mice (Fig. S3L and S3M). YH2AX staining pattern of WT meiocytes and oocytes in primordial and mature follicles resembled those reported previously (Yamaguchi et al., 2012). These data suggest important role of Tet2 in DNA repair of early germ cells and oocytes.

The quality of mature oocytes is a major determinant of reproductive capacity and fertility of females. We assessed the number and morphology of oocytes collected from mice at different age $16-17 \mathrm{~h}$ following superovulation treatment. The number of oocytes decreased with age in WT females, well-recognized phenomenon in reproductive aging, and also in $\mathrm{Tet}^{-/-}$female mice (Fig. 1B and $1 \mathrm{C}$ ). The number of oocytes obtained from Tet2 $2^{-l-}$ mice did not differ from that of WT females regardless of age, and this corroborated similar number of follicles shown earlier (Fig. S1I and S1J). However, polar body extrusion was notably suppressed or delayed in $T e t 2^{-1-}$ oocytes and this became more evident with age, unlike WT oocytes (Fig. 1B and 1C). Furthermore, the frequency of abnormal or disrupted spindles and chromosome misalignment at metaphase was increased in Tet2 ${ }^{-/-}$ oocytes from mice at different age (Fig. 1D and 1E). Consequently, rate of development to blastocysts of Tet2 ${ }^{-/-}$oocytes following in vitro fertilization (IVF) was lower than that of WT, and further declined with age (Fig. $1 F$ and $1 G$ ). While successfully fertilized WT oocytes cleaved and reached blastocysts by $72 \mathrm{~h}$ in culture, most of Tet2 ${ }^{-/-}$oocytes and oocytes from old WT mice still were at morula stage, indicative of delayed embryo development. In addition, blastocysts 
developed from Tet2 ${ }^{-/-}$oocytes were smaller, and the percentage of hatching blastocysts also was lower at young and old age, compared to that of age-matched WT controls (Fig. 1F and 1G). These data showed that Tet ${ }^{-1-}$ deficiency impairs polar body extrusion, spindle structure and chromosome alignment, reducing oocyte quality and subsequent embryo development.

The spindle assembly checkpoint (SAC) safeguards correct chromosome alignment by sensing unattached kinetochores and delaying anaphase onset (London and Biggins, 2014). Mad2 and Cenpe, as a bridge to connect kinetochore and microtubule, would be removed from kinetochores $8 \mathrm{~h}$ post germinal vesicle breakdown (GVBD) (Marangos et al., 2015). We wondered whether the two proteins were implicated in reduced polar body extrusion in Tet $2^{-1-}$ oocytes. More strongly stained Mad2 and Cenpe signals at kinetochores were revealed in Tet2 $2^{-1-}$ oocytes $8 \mathrm{~h}$ post GVBD, compared to WT oocytes (Fig. S4A-D). Moreover, by careful examination of spindles and chromosome morphology of oocytes 16-17 $\mathrm{h}$ after superovulation, we observed apparently more abnormal oocytes (about $60 \%$ ), which were at $\mathrm{MI}$, anaphase or telophase stage with misaligned chromosomes or disrupted spindles in Tet2 ${ }^{-/-}$mice and these abnormalities increased to nearly $80 \%$ at old age, whereas a majority (about $80 \%$ ) of oocytes from young WT mice displayed normal meiosis with appropriate chromosome alignment at the metaphase spindle (Fig. S4E and $\mathrm{S} 4 \mathrm{~F}$ ). The delayed MI progression is linked to postponed or suppressed polar body extrusion. Thus, Tet2 is implicated in the checkpoint control during chromosome congregation and separation.

To understand the molecular bases of the declined reproductive capacity and associated meiotic defects caused by Tet2 deficiency shown above, we performed transcriptome analysis by single-cell RNA-seq of WT and Tet2 ${ }^{-/}$MIl oocytes collected from young and old mice (Fig. 2A). Based on the Principal Component Analysis (PCA), the distributions of two PCA components differed among WT and Tet2 ${ }^{-/-}$oocytes at young and old age, and some overlapped between young $\mathrm{Tet}^{-1-}$ and old WT oocytes. Distribution of PCA in old Tet2 $2^{-/-}$oocytes was more complicated, suggesting that Tet2 deficiency and aging jointly affected the oocyte transcription (Fig. 2A). Differentially expressed genes (DEGs) were obtained by Deseq2 and genes highly expressed in different groups were compared by pheatmap. Genes important for meiosis Sycp3 and Dmc1, Wapl, required for accurate meiosis I chromosome segregation and controlling SAC, Mad2/1, Mad1/1 and Zfp207 for chromosome alignment during meiosis I, and Fbxw7, Ythdf2, critical for oocyte maturation and competence, were highly expressed in young WT oocytes but downregulated in oocytes from other three groups (Fig. 2B). Moreover, we performed GO and KEGG enrichment using KOBAS database with the adjusted $P$-value (FDR $<0.05$, by statistical algorithm of Benjamini and Hochberg). Genes highly expressed in young WT oocytes but downregulated in
Figure 2. Differential gene expression of $\mathrm{Tet2}^{-/}$and WT oocytes. (A) PCA analysis of young WT ( $n=24)$, Tet2 $^{-1-}(n=16)$, old WT $(n=22)$ and Tet ${ }^{-1-}(n=16)$ oocytes using the CPM for all genes from 2-3 mice. (B) Pheatmap showing differential gene expression of young WT, Tet $2^{-1-}$, old WT and Tet2 $2^{-1-}$ oocytes. (C) Barplot showing the enrichment of genes highly expressed in young WT oocytes but lowly expressed in other groups. $\mathrm{X}$-axis represents the corrected p-value (FDR) using Benjamini and Hochberg. (D) Pheatmap showing genes enriched in actin cytoskeleton after Tet2 knockout. (E) Venn diagram of upregulated and downregulated genes in young and old Tet $2^{-1-}$ oocytes, compared with WT oocytes. (F) Pheatmap showing genes upregulated and downregulated in both $\mathrm{Tet}^{-/-}$young and old oocytes.

(G) Bar plot illustrating the enrichment of genes upregulated or downregulated after Tet2 knockout. X-axis represented the corrected p-value (FDR) using Benjamini and Hochberg. ( $\mathrm{H})$ Representative images of oocytes costained with $\mathrm{F}$-actin and $\alpha$-Tubulin by immunofluorescence microscopy. Scale bar, $10 \mu \mathrm{m}$. Right panel, Relative expression F-actin level by quantification of immunofluorescence intensity averaged from various oocytes using Image J $(n=5-7)$.

other three groups were enriched in cellular response to DNA damage stimulus, DNA repair, sister chromatid segregation, meiosis I cell cycle process and meiotic cell cycle (Fig. 2C). KEGG analysis also showed that highly expressed genes in young WT group were enriched in oocyte meiosis, cell cycle, and metabolic pathways. The transcriptome data suggested that Tet deficiency reduces DNA repair and meiosis progression and SAC, leading to accelerated decline in oocyte competence with age.

Moreover, we looked at transcriptional changes in oocytes during natural aging, to understand the reduced quality of oocytes. Aging resulted in differential expression of genes, including downregulation of Id3, Sycp3, Dmc1, and Sycp1, and upregulation of Atm, Rad50, and Brca2 (Fig. S5A). t-SNE showed distribution in the gene expression between oocytes of young and old mice (Fig. S5B). By signaling pathway analysis, genes downregulated were enriched in oocyte meiosis and maturation and regulation of actin cytoskeleton, but genes related to Alzheimer's disease were upregulated (Fig. S5C and S5D). By functional enrichment (GO) analysis, genes related to methylation were upregulated, but genes involved in reciprocal meiotic recombination, demethylation, sister chromatid cohesion, DNA repair and embryo development were downregulated in old WT oocytes (Fig. S5E and S5F). These molecular defects in DNA repair, oocyte maturation, spindle and sister chromatid cohesion are consistent with declined function of oocytes with age shown previously. 


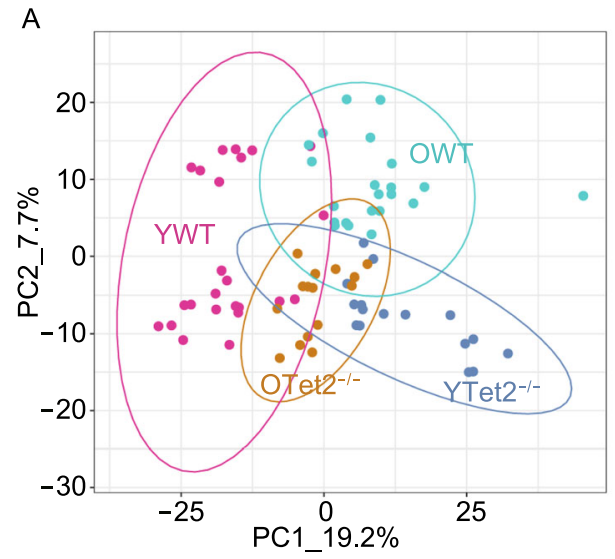

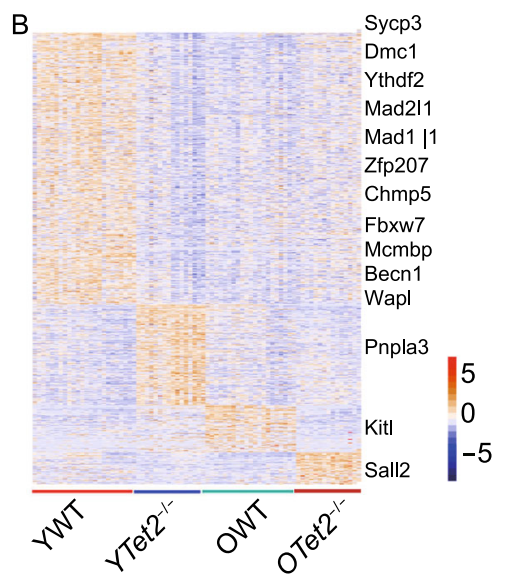
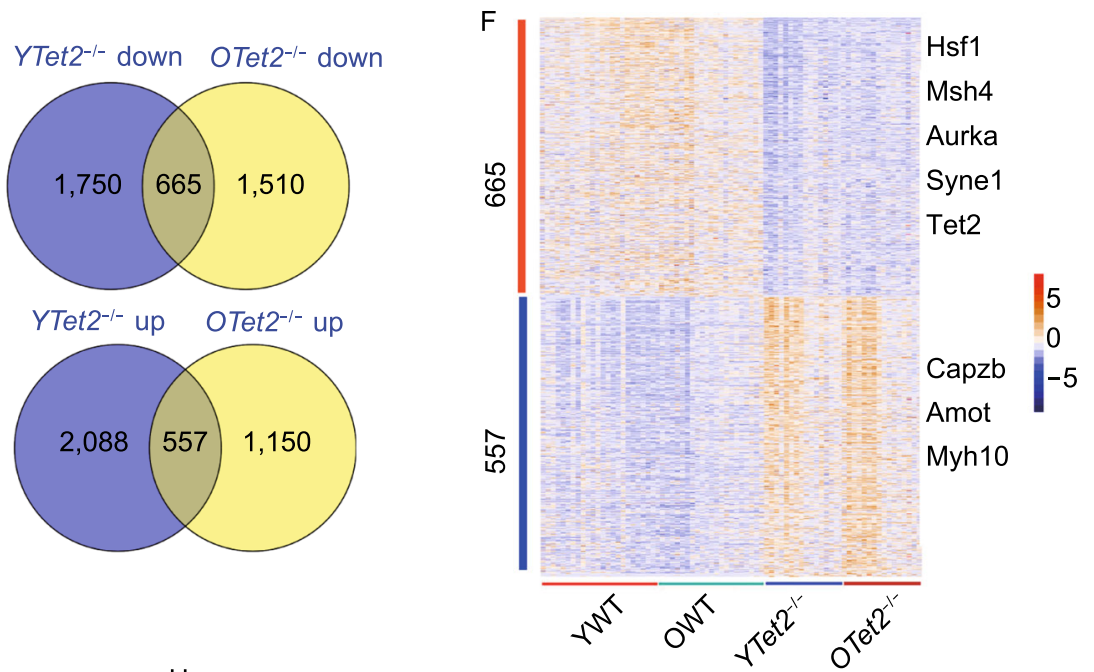

$\mathrm{H}$
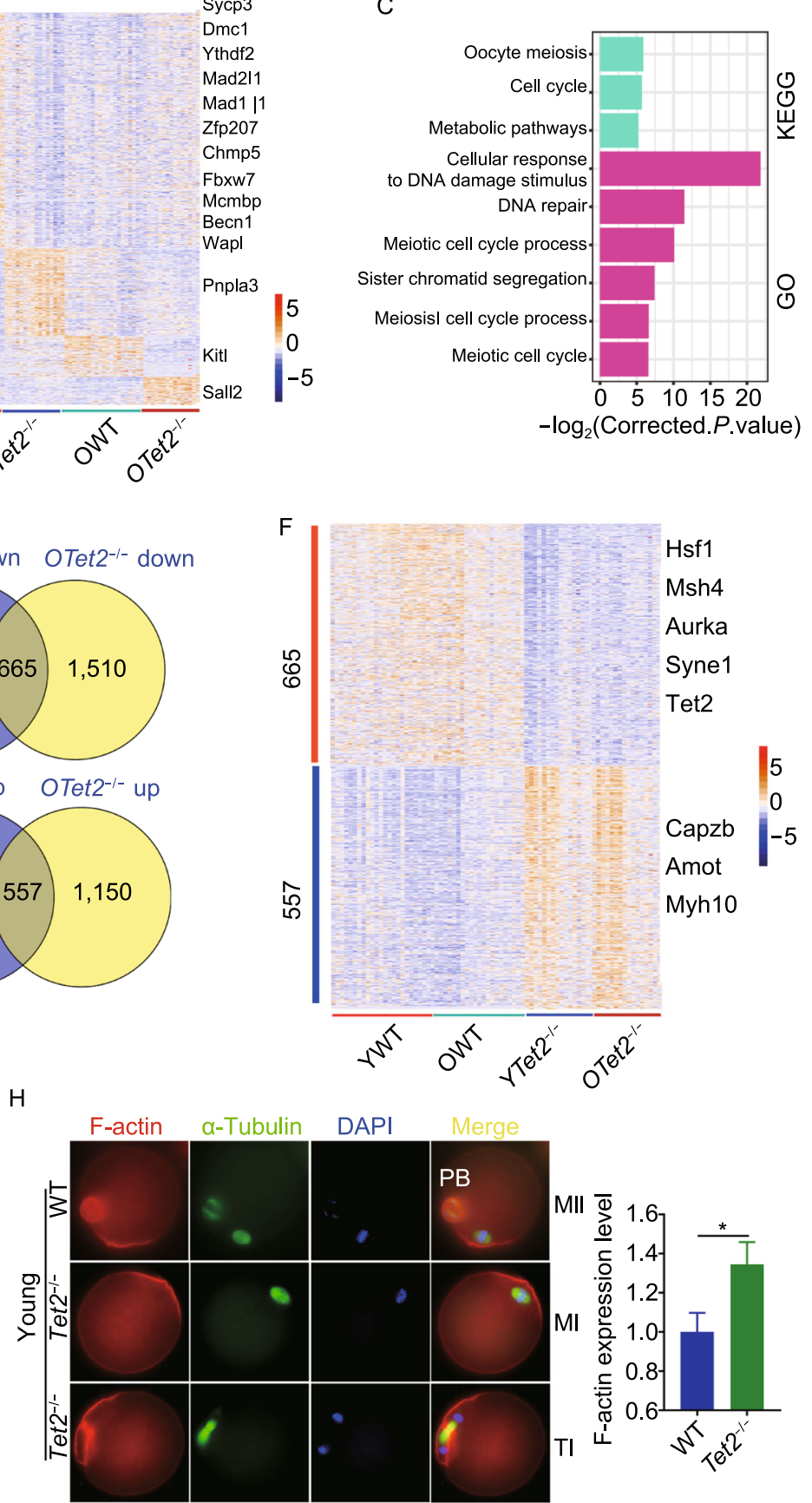

E

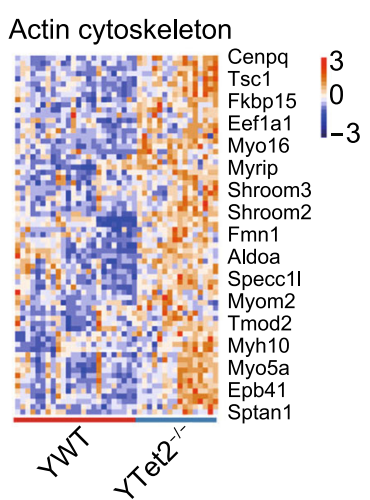

G

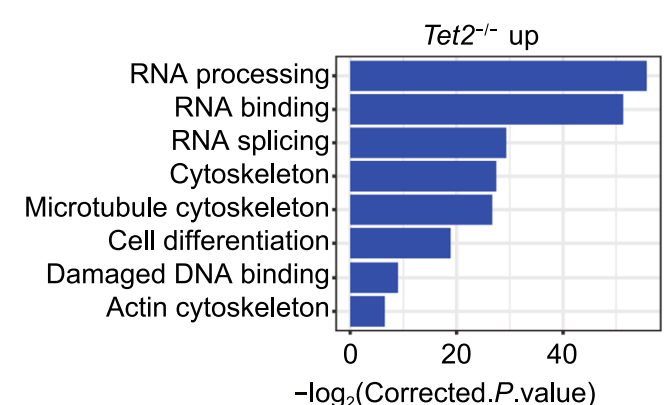

。

$-\log _{2}$ (Corrected.P.value)

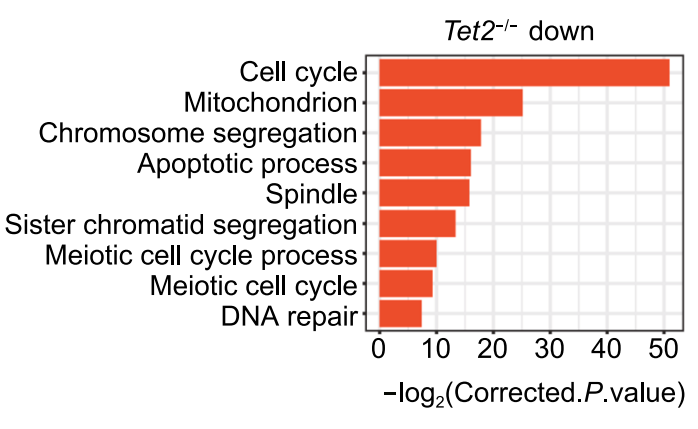

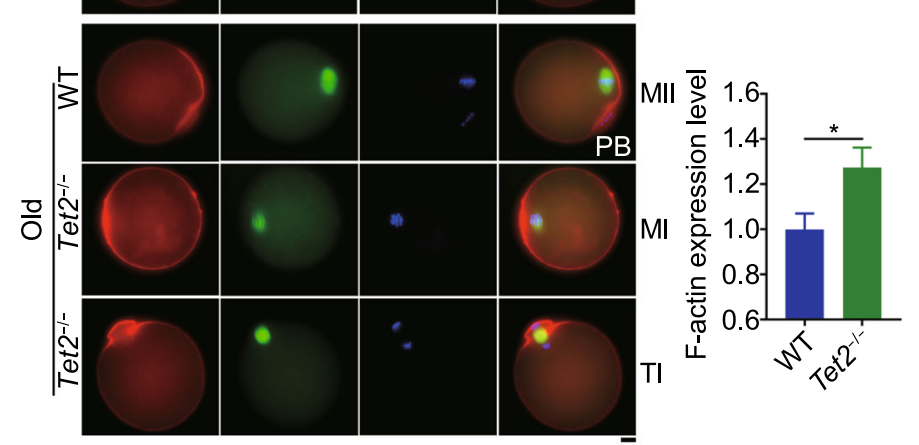


We further analyzed single-cell transcriptome data of Tet ${ }^{-/-}$and WT oocytes ( $n=16$ and 24, respectively) collected from 2-3 mice at young age. Upregulated genes in Tet $2^{-1-}$ oocytes surprisingly were enriched in actin binding and actin cytoskeleton (Fig. 2D). We also compared Tet2 ${ }^{-1-}$ with WT oocytes from young and old mice. Venn diagram revealed 665 genes co-downregulated and 557 genes coupregulated after Tet2 loss (Fig. 2E). As expected, Tet2 was downregulated in Tet2 ${ }^{-/-}$compared with WT oocytes. In addition, genes for spindle assembly checkpoint and DNA repair such as Hsf1, Msh4, Aurka, and Syne1, were downregulated while genes related with actin cytoskeleton organization, including Myh10, Captz and Amot, were among the highest upregulated in Tet2 $2^{-/-}$oocytes (Fig. 2F). Upregulated genes after Tet2 knock-out also included RNA processing and splicing, cell differentiation, damaged DNA binding and actin cytoskeleton (Fig. 2G). Genes downregulated were enriched in cell cycle, mitochondrion, apoptotic process, spindle, sister chromatid segregation, meiotic cell cycle process and DNA repair (Fig. 2G).

Genes associated with increased DNA damage, aberrant upregulation of actin cytoskeleton and downregulated spindle checkpoint of Tet2 ${ }^{-l-}$ oocytes got our attention, and these defects together likely link to suppression or delay of polar body extrusion resulting from Tet2 deficiency. To substantiate the transcriptome results, we performed immunostaining of F-actin filament in oocytes. Indeed, normal MII oocytes of WT mice exhibited polar body extrusion enclosed within actin ring, and well separated chromosomes surrounded by actin filament residue (Fig. $2 \mathrm{H}$ ). However, stronger actin filament immunofluorescence signal was found in oocytes of Tet2 ${ }^{-/-}$mice, compared to WT mice. Distribution of more uniformly stained actin filaments in Tet $2^{-/-}$oocytes coincided with $\mathrm{MI}$ arrest or anaphase or telophase (TI) without clear polar body separation, or with delayed polar body extrusion (Fig. $2 \mathrm{H}$ ). Aberrantly distributed actin at the high expression level likely disrupts asymmetry division of oocyte and polar body extrusion. Hence, Tet2 is required for normal meiosis progression by regulating SAC and actin cytoskeleton.

As age also alters DNA methylation and Tet enzymes participate in DNA demethylation, we performed low-input methylation sequencing on MII oocytes to look at potential changes in methylome caused by Tet2 deficiency and natural aging. Global DNA methylation level increased in Tet2 ${ }^{-1-}$ oocytes, compared with young WT oocytes, and also slightly increased in natural aging/old WT oocytes, despite no statistical differences (Fig. S6A). In comparison of the differentially methylated genes of $T e t 2^{-1-}$ with those of natural aging, old WT oocytes, about half of hypomethylated genes in old WT oocytes (total 693 hypomethylated genes) were hypomethylated in Tet2 ${ }^{-/-}$oocytes (total 602 hypomethylated genes). About one third of hypermethylated genes in natural aging (total 663 hypermethylated genes) also were hypermethylated (237) after Tet2 deficiency (total 498 hypermethylated genes) (Fig. S6B). These data suggest that Tet2 ${ }^{-/-}$and old WT oocytes (or natural aging oocytes) share 1/3-1/2 DNA methylation modifications. Analysis of genomewide methylation levels revealed that gene body regions were slightly hypermethylated in young Tet2 $2^{-/-}$and old WT oocytes, and Tet2 $2^{-1-}$ oocytes had relatively lower methylation levels at TSS and TES, compared with those of young and old WT oocytes (Fig. S6C).

Moreover, in old WT oocytes, hypomethylated genes were involved in aging signaling pathway, and hypermethylated genes enriched in regulation of transcription and actin cytoskeleton signaling pathways (Fig. S6D), implying that these signaling pathways may be down-regulated. Indeed, transcriptome data showed that actin cytoskeleton signaling pathway was declined in old WT oocytes (Fig. S5C). Genes involved in regulation of transcription were hypermethylated in both Tet2 $2^{-1-}$ and old WT oocytes (Fig. S6D), indicating that Tet $2^{-/-}$and old oocytes both possibly negatively regulate transcriptional activity. On the other hand, genes involved in cytoskeleton pathways were hypomethylated and coincidently these genes were highly expressed in Tet2 ${ }^{-1-}$ oocytes, while they were hypermethylated in old WT oocytes (Fig. S6D). Promoter methylation levels of actin cytoskeleton were reduced in Tet2 ${ }^{-/-}$oocytes, but not in old WT oocytes (Fig. S6E). Yet, promoter methylation levels of genes enriched in metabolic process were higher in Tet2 $2^{-1-}$ and old WT oocytes (Fig. S6F). Promoter methylation levels of genes enriched in sister chromatid segregation (GO: 0000819), spindle checkpoint (GO: 0031577), DNA repair (GO: 0006281) and cellular response to DNA damage (GO: 0006974) were higher in young Tet2 ${ }^{-/-}$and old WT oocytes compared with young WT oocytes (Fig. S6F). We also analyzed methylation levels at the enhancers of the same genes and showed that the methylation levels at the enhancers generally were higher, but did not show significant changes except for higher levels at DNA repair genes in Tet2 ${ }^{-/-}$oocytes than in WT oocytes (Fig. S6G). Overall, Tet2deficient and natural aging oocytes exhibit similarities in methylation modifications at some regions, but differ also in others, for instance, in the methylation of actin cytoskeleton signaling pathway.

Additionally, Tet proteins themselves also have been found to regulate enhancer activity (Lu et al., 2014). We also attempted to look at the potential regulation of gene expression changes using ChIP data reported in ESCs (Hon et al., 2014). Tet2 deletion resulted in increased $5 \mathrm{hmC}$ binding to the gene body. We further analysed the $5 \mathrm{hmC}$ binding of genes in actin cytoskeleton and $5 \mathrm{hmC}$ binding indeed was increased after Tet2 knockdown (Fig. S7A), although global $5 \mathrm{hmC}$ levels were generally reduced in Tet2 knockdown compared with WT controls (Fig. S7B). H3K27ac binding signal at these genes enriched in actin cytoskeleton was noticeably increased in Tet2 ${ }^{-/-}$oocytes but H3K27me3 showed only slight changes and the binding signal was 
much lower than that of H3K27ac (Fig. S7C). It appears that increased expression of genes related to actin cytoskeleton coincided with elevated H3K27ac enrichment. But, histone modifications in oocytes and ESCs may be not the same, and these regulations require further investigation.

Together, the whole knockout mouse model demonstrated that Tet2 deficiency decreases oocyte development and quality, and accelerates age-associated infertility. The in vitro embryo developmental delay and incompetence is consistent with reduced embryo development in vivo, supporting the accelerated infertility with mouse age. Tet2 and its mediated DNA methylation changes play critical role in germ cells at various developmental stages. Tet1 has an important role in meiotic gene activation at entry into prophase I in the embryonic ovary and Tet 1 deficiency significantly reduces oocyte numbers and fertility by increased apoptosis (Yamaguchi et al., 2012). We show that effects of Tet2 on meiosis prophase I appear to be less, relative to the function of Tet1 in embryonic gonads. Possible compensatory effects of Tet 1 and Tet 3 in the Tet2-deficient mice also cannot be excluded. Tet2 deficiency did not directly elevate apoptosis, and this may allow the defective germ cells with aberrant meiosis progression to survive.

Tet2 and its role in regulating DNA methylation levels are involved in meiosis, DNA repair, actin cytoskeleton and spindle assembly checkpoint, chromosome alignment and segregation, and thus ploidy of oocytes. Our data suggests that promoter hypermethylation resulting from Tet2-deficiency specifically regulates genes in meiosis cell cycle, spindle assembly checkpoint and DNA repair, and the aberrantly increased enhancer activity may contribute to elevated actin cytoskeleton signaling. It is possible that both DNA methylation and enhancer activity by Tet-mediated DNA demethylation regulate specific genes. Indeed, Tet-mediated DNA demethylation mainly occurs at distal regulatory enhancer elements/regions, and enhancers are generally hypomethylated when they are bound by cell type-specific transcription factors (Lu et al., 2014). Hypermethylation can be found at enhancers (H3K4me1 and H3K27ac) of some genes and hypomethylation also can be found at enhancers of other genes depending on their regulatory elements and heterochromatin marks in these regions (Lu et al., 2014). Also, we cannot exclude the possibility that other factors may indirectly affect oocyte quality by Tet 2 deficiency and reduced $5 \mathrm{hmC}$ levels in other somatic cells, including bone marrow defects. Nevertheless, the fertility defects in Tet2 ${ }^{-/-}$mice mainly result from defective meiotic progression and thus declined quality of oocytes. In future experiments, germline specific conditional knockout of Tet2 can be created to confirm this observation. These findings broaden our understanding of the role of Tet enzyme in regulating fertility and reproductive aging. Notably, Tet2 deficiency results in aberrant activation of actin cytoskeleton that impedes asymmetry division of oocytes, suggesting important roles of Tet2 in regulating cell division. Defective asymmetry cell division by Tet2 mutation or deficiency also may be implicated in somatic stem cell aging.

\section{FOOTNOTES}

We thank Wei Deng for initial help in the experiments, and Lizhi Yi and Jiang Liu from Beijing Institute of Genomics for helping DNA methylation experiment. This work was supported by the National Natural Science Foundation of China (Grant Nos. 31571546 and 91749129).

H.W. performed major experiments and prepared the manuscript. L.L.L. analyzed the RNA-seq data and prepared the manuscript. M. $G$ conducted oocyte library construction and analysis for methylome. G.H., J.Y., H.W. and W.D. helped genotyping and other tests. C.T. helped IVF experiments. G.X. provided materials, advised the project, and revised the manuscript. L.L. conceived the study, designed experiments and wrote the manuscript.

Huasong Wang, Linlin Liu, Mo Gou, Guian Huang, Chenglei Tian, Jiao Yang, Haiying Wang, Qin Xu, Guo_Liang Xu and Lin Liu declare that they have no conflict of interest.

All institutional and national guidelines for the care and use of laboratory animals were followed.

Huasong Wang ${ }^{1,2}$, Linlin $\mathrm{Liu}^{1,2}$, Mo Gou ${ }^{1,2}$, Guian Huang ${ }^{1,2}$, Chenglei Tian ${ }^{1,2}$, Jiao Yang ${ }^{1,2}$, Haiying Wang ${ }^{1,2}$, Qin $X u^{3}$, Guo_Liang $\mathrm{Xu}^{3,4}$, Lin Liu ${ }^{1,2 \bowtie}$

${ }^{1}$ Department of Cell Biology and Genetics, College of Life

Sciences, Nankai University, Tianjin 300071, China

2 State Key Laboratory of Medicinal Chemical Biology, Nankai University, Tianjin 300071, China

${ }^{3}$ State Key Laboratory of Molecular Biology, Shanghai Institute of Biochemistry and Cell Biology, Chinese Academy of Sciences, Shanghai 200031, China

${ }^{4}$ Key Laboratory of Medical Epigenetics, Institutes of Biomedical

Sciences, Fudan University, Shanghai 200032, China

$\triangle$ Correspondence: liulin@nankai.edu.cn (L. Liu)

Accepted November 4, 2020

\section{OPEN ACCESS}

This article is licensed under a Creative Commons Attribution 4.0 International License, which permits use, sharing, adaptation, distribution and reproduction in any medium or format, as long as you give appropriate credit to the original author(s) and the source, provide a link to the Creative Commons licence, and indicate if changes were made. The images or other third party material in this article are included in the article's Creative Commons licence, unless indicated otherwise in a credit line to the material. If material is not included in the article's Creative Commons licence and your intended use is not permitted by statutory regulation or exceeds 
the permitted use, you will need to obtain permission directly from the copyright holder. To view a copy of this licence, visit http:// creativecommons.org/licenses/by/4.0/.

\section{REFERENCES}

Cimmino L, Dolgalev I, Wang Y, Yoshimi A, Martin GH, Wang J, Ng V, Xia B, Witkowski MT, Mitchell-Flack M et al (2017) Restoration of TET2 function blocks aberrant self-renewal and leukemia progression. Cell 170(1079-1095):e1020

Dawlaty MM, Breiling A, Le T, Raddatz G, Barrasa MI, Cheng AW, Gao Q, Powell BE, Li Z, Xu M et al (2013) Combined deficiency of Tet1 and Tet2 causes epigenetic abnormalities but is compatible with postnatal development. Dev Cell 24:310-323

Delhommeau F, Dupont S, Della Valle V, James C, Trannoy S, Masse A, Kosmider O, Le Couedic JP, Robert F, Alberdi A et al (2009) Mutation in TET2 in myeloid cancers. N Engl $\mathrm{J}$ Med 360:2289-2301

Ferrone CK, Blydt-Hansen M, Rauh MJ (2020) Age-associated TET2 mutations: common drivers of myeloid dysfunction. Cancer and cardiovascular disease. Int J Mol Sci 21:626

Hon GC, Song CX, Du T, Jin F, Selvaraj S, Lee AY, Yen CA, Ye Z, Mao SQ, Wang BA et al (2014) $5 \mathrm{mC}$ oxidation by Tet 2 modulates enhancer activity and timing of transcriptome reprogramming during differentiation. Mol Cell 56:286-297

Horvath S, Raj K (2018) DNA methylation-based biomarkers and the epigenetic clock theory of ageing. Nat Rev Genet 19:371-384
Huang G, Liu L, Wang H, Gou M, Gong P, Tian C, Deng W, Yang J, Zhou TT, Xu GL et al (2020) Tet1 deficiency leads to premature reproductive aging by reducing spermatogonia stem cells and germ cell differentiation. iScience 23:100908

Ko M, Huang Y, Jankowska AM, Pape UJ, Tahiliani M, Bandukwala HS, An J, Lamperti ED, Koh KP, Ganetzky R et al (2010) Impaired hydroxylation of 5-methylcytosine in myeloid cancers with mutant TET2. Nature 468:839-843

London N, Biggins S (2014) Signalling dynamics in the spindle checkpoint response. Nat Rev Mol Cell Biol 15:736-747

Lu F, Liu Y, Jiang L, Yamaguchi S, Zhang Y (2014) Role of Tet proteins in enhancer activity and telomere elongation. Genes Dev 28:2103-2119

Marangos P, Stevense M, Niaka K, Lagoudaki M, Nabti I, Jessberger $\mathrm{R}$, Carroll J (2015) DNA damage-induced metaphase I arrest is mediated by the spindle assembly checkpoint and maternal age. Nat Commun 6:8706

Moran-Crusio K, Reavie L, Shih A, Abdel-Wahab O, Ndiaye-Lobry D, Lobry C, Figueroa ME, Vasanthakumar A, Patel J, Zhao X et al (2011) Tet2 loss leads to increased hematopoietic stem cell selfrenewal and myeloid transformation. Cancer Cell 20:11-24

Saitou M, Miyauchi H (2016) Gametogenesis from Pluripotent Stem Cells. Cell Stem Cell 18:721-735

Wu X, Zhang Y (2017) TET-mediated active DNA demethylation: mechanism, function and beyond. Nat Rev Genet 18:517-534

Yamaguchi S, Hong K, Liu R, Shen L, Inoue A, Diep D, Zhang K, Zhang $Y$ (2012) Tet1 controls meiosis by regulating meiotic gene expression. Nature 492:443-447
Huasong Wang, Linlin Liu, and Mo Gou—Equal contributions.

Electronic supplementary material The online version of this article (https://doi.org/10.1007/s13238-020-00805-8) contains supplementary material, which is available to authorized users. 